\title{
Association of Hyperhomocysteinemia and Adverse Postoperative Outcomes of on-Pump Primary Isolated Coronary Artery Bypass Grafting Surgery among Postmenopausal Women
}

\author{
Ahmed Saber, M.D, FRCS (Glasg) ${ }^{1 *}$, Ayat Fathy, M.Sc ${ }^{2}$ and Sherif Nasr, M.D ${ }^{3}$ \\ ${ }^{1}$ Department of Cardiothoracic Surgery, Cairo University, Egypt \\ ${ }^{2}$ Department of Obstetrics and Gynecology, TCH, Ministry of Health, Egypt \\ ${ }^{3}$ Department of Cardiothoracic Surgery, El-Fayoum University, Egypt
}

\begin{abstract}
Background and objectives: Studies investigating the possible role of hyperhomocysteinemia $(\mathrm{HHC})$ in increasing postoperative morbidity and mortality among postmenopausal women undergoing on-pump primary isolated coronary artery bypass grafting (CABG) surgery are sparse in the literature. The knowledge about the relationship of preoperative $\mathrm{HHC}$ and postoperative adverse cardiovascular events is not adequately covered. This study is initiated to ascertain the hypothesized higher prevalence of HHC in postmenopausal women with ischemic heart disease (IHD) scheduled for onpump primary isolated CABG and tracing its association to the proposed adverse postoperative complications especially thromboembolic ones linked in the literature to HHC.
\end{abstract}

Methods: This prospective comparative study included 77 postmenopausal women who presented with IHD requiring primary surgical myocardial revascularization (on-pump isolated primary CABG surgery). The study was conducted in the period between January 2016 and January 2019. The study population was divided into two groups based on their homocysteine (HCY) level. Group (I) included 34 patients with $\mathrm{HCY}$ values more than $15 \mathrm{micro} . \mathrm{mol} / \mathrm{L}$ and group (II) included 43 patients with $\mathrm{HCY}$ values less than $15 \mathrm{micro}$.mol/L. The study population included patients having left main or left main-equivalent coronary artery disease, multi-vessel coronary artery disease, and one or two coronary artery disease. We excluded patients scheduled for off-pump CABG, patients with associated pathologies such as ascending aortic aneurysm/dissection, left ventricular aneurysm, and ventricular septal defects. Patients with concomitant valvular heart disease (including ischemic mitral regurgitation requiring intervention), malignancy, multiple co-morbidities e.g. liver cell failure, renal failure, respiratory failure, and patients with poor left ventricular ejection fraction (LVEF $<40 \%)$ were also excluded. The postoperative studied variables included ICU parameters (duration of mechanical ventilation, duration of inotropic support, total blood loss, blood glucose level, total duration of ICU stay), operative mortality, total hospital stay, and adverse complications (myocardial infarction, cerebrovascular accidents, pulmonary embolism, peripheral arterial/venous thromboembolism, low cardiac output syndrome, rhythmic complications, hemorrhagic complications, respiratory complications, acute renal failure, deep and superficial wound infections), overall hospital complications and overall one-year mortality and survival.

Results: Mean age was $64.34 \pm 5.81$ years in group (I) and $60.95 \pm 6.54$ years in group (II). Mean serum HCY level was $18.55 \pm 1.11 \mathrm{micro} . \mathrm{mol} / \mathrm{L}$ (range 15.50-23.6) for group (I) and $9.83 \pm 1.03 \mathrm{micro} . \mathrm{mol} / \mathrm{L}$ (range 5.6-12.7) for group (II). Group (I) showed statistically significant differences $(p<0.05)$ regarding preoperative variables (more previous $\mathrm{MI}$ attacks, more CCU admissions, lower LVEF\%, higher EurOSCORE, more peripheral vascular disease and surgeries, more previous cerebrovascular accidents, more number of affected coronary vessels and higher peak serum creatinine levels) and postoperative variables (duration of mechanical ventilation, period of ICU stay, total duration of hospital stay, low cardiac output syndrome, IABP insertion, cerebrovascular accidents, acute renal failure and peak serum creatinine level). However, the overall hospital complication rate was $12(35.29 \%)$ and $13(30.23 \%)$ for group (I) and (II) respectively $(p>$ $0.05)$. During the follow-up period, both groups expressed comparable results with no statistical significance, the overall one-year survival was $94.11 \%$ and $95.34 \%$ in group (I) and (II) respectively $(p>0.05)$ and the overall mortality was 4 (5.19\%) (two deaths from each group; one in-hospital, and one during the follow-up period) $(p>0.05)$.

Conclusion: HHC was found to have high prevalence among postmenopausal women having IHD and undergoing CABG surgery. It was found to be associated with adverse postoperative complications as well as preoperative thromboembolic and cardiovascular insults. On-pump CABG aggravates the thrombotic potentiality in these patients. However, operative and postoperative mortality as well as one-year survival were not associated with HHC. Preoperative assay of $\mathrm{HCY}$ level and HHC stratification in the reviewed pre-CABG risk factors are recommended. Preoperative supplementation with folic acid, vitamin B12 and vitamin B6 may play a role in lowering the preoperative $\mathrm{HCY}$ level, thus, lowering the proposed postoperative adverse outcomes. Therapeutic strategies such as preoperative aggressive treatment with antiplatelet or anticoagulant drugs may also be tried to reduce the thrombogenic risks for this subset of patients.

Copyright: (c) 2019 Saber A, et al. This is an open-access article distributed under the terms of the Creative Commons Attribution License, which permits unrestricted use, distribution, and reproduction in any medium, provided the original author and source are credited. 
Citation: Saber A, Fathy A, Nasr S (2019) Association of Hyperhomocysteinemia and Adverse Postoperative Outcomes of on-Pump Primary Isolated Coronary Artery Bypass Grafting Surgery among Postmenopausal Women. Ann Heart 4(1):86-95

\section{Keywords}

Hyperhomocysteinemia, HHC, Homocysteine, HCY, Primary CABG, Postmenopausal

\section{Introduction}

Homocysteine (HCY) is a sulfur-containing amino acid derived from methionine. Its metabolism involves remethylation, transsulfuration, and demethylation processes, which require folic acid, vitamin B12, and vitamin B6 [1]. Hyperhomocysteinemia $(\mathrm{HHC})$ is defined as a plasma level of $\mathrm{HCY}$ exceeding 15 micro.mol/L [2].

Whereas $30-50 \%$ of the variation in cardiovascular disease occurrence remains unexplained by the known traditional risk factors such as smoking, hypercholesterolemia, hypertension, and diabetes mellitus $[3,4]$, experimental and epidemiological based-evidence suggests that $\mathrm{HHC}$ is an independent risk factor for the development of atherosclerosis, thrombosis, and other cardiovascular diseases, which may be attributed to impaired fibrinolysis or increased platelet reactivity [5-7]. Moreover, evidence exists supporting significant impact of HHC on endothelial resistance to thrombosis and vasodilatory and antithrombotic effects of nitric oxide, accounting for the increased risks of thromboembolic events in patients with cardiovascular disease [8] .

Basal homocysteinemia was found to be significantly higher in men than women [9]. After menopause, it increases significantly in women, approaching those in men. This is most probably due to estrogen deficiency because in young woman where estrogen production is high, serum lipids and $\mathrm{HCY}$ levels are normal [10]. But after menopause, abnormal lipid profile and $\mathrm{HHC}$ and increase incidence of coronary heart disease show a possible relationship among estrogen, normal lipid profile, normal HCY levels and relative immunity to coronary heart disease [11]. Given that the postmenopausal status has been shown to be associated with increased HCY levels, it is conceivable that such an increase could have a role in the augmented cardiovascular morbidity of postmenopausal women [12].

Patients with $\mathrm{HHC}$ undergoing cardiac surgery (especially $C A B G$ ) on cardiopulmonary bypass (CPB) which represents a prothrombotic state itself are at significant risk for serious adverse mesenteric ischaemia, stroke, pulmonary embolism, and deep vein thrombosis, resulting in high operative morbidity and mortality [13-15].

Studies investigating the possible role of $\mathrm{HHC}$ in increasing postoperative morbidity and mortality among postmenopausal women undergoing primary isolated CABG are sparse in the literature. The knowledge about the relationship of preoperative $\mathrm{HHC}$ and postoperative adverse cardiovascular events is not adequately covered. This study is initiated to trace and investigate the possible association between $\mathrm{HHC}$ and the proposed adverse postoperative complications (especially thromboembolic ones linked in the literature to $\mathrm{HHC}$ ) and mortality and also to ascertain the hypothesized higher prevalence of $\mathrm{HHC}$ in postmenopausal women with ischemic heart disease (IHD) scheduled for primary isolated CABG.

\section{Patients and Methods}

\section{Study design and patient population}

This prospective comparative study included 77 postmenopausal women who presented with IHD requiring primary surgical myocardial revascularization (on-pump primary isolated CABG surgery). The study was conducted after obtaining the approval of the ethical committee of the department of Cardiothoracic Surgery, Faculty of Medicine, Cairo University; and after a written informed consent from each and every participant for the scientific treatment. All the surgeries were carried out in Egypt (conducted in operating theatre of the department of Cardiothoracic Surgery, Faculty of Medicine, Cairo University; and other open heart surgery centers) using standard open heart surgical procedures. The study was conducted in the period between January 2016 and January 2019. Surgical and medical treatments were at the discretion of surgical team in accordance with evidence-based guidelines, with no specific treatment mandated or new intervention applied as a part of this study. The study population was divided into two groups based on their HCY level. Group (I) included 34 patients with $\mathrm{HCY}$ values more than 15 micro. $\mathrm{mol} / \mathrm{L}$ and group (II) included 43 patients with $\mathrm{HCY}$ values less than 15 micro.mol/L. The data of the study population was collected from the cardiothoracic section computer database supplemented by a review of hospital records. All the data were studied and thoroughly evaluated in the preoperative, intraoperative and postoperative periods (for one year follow-up duration).

\section{Inclusion and exclusion criteria}

The study population included postmenopausal women scheduled for elective on-pump (requiring cardiopulmonary bypass "CPB") primary isolated CABG surgery presenting with IHD having left main or left main-equivalent coronary artery disease, multi-vessel coronary artery disease, and one or two coronary artery disease. We excluded patients scheduled for off-pump CABG, patients with associated pathologies such as ascending aortic aneurysm/dissection, left ventricular aneurysm, and ventricular septal defects. Patients with concomitant valvular heart disease (including ischemic mitral regurgitation requiring intervention), malignancy, multiple co-mor-

*Corresponding author: Ahmed Saber, M.D, FRCS (Glasg), Department of Cardiothoracic Surgery, Cairo University, Cairo, Egypt

Accepted: June 20, 2019

Published online: June 22, 2019

Citation: Saber A, Fathy A, Nasr S (2019) Association of Hyperhomocysteinemia and Adverse Postoperative Outcomes of on-Pump Primary Isolated Coronary Artery Bypass Grafting Surgery among Postmenopausal Women. Ann Heart 4(1):86-95 
Citation: Saber A, Fathy A, Nasr S (2019) Association of Hyperhomocysteinemia and Adverse Postoperative Outcomes of on-Pump Primary Isolated Coronary Artery Bypass Grafting Surgery among Postmenopausal Women. Ann Heart 4(1):86-95

bidities e.g. liver cell failure, renal failure, respiratory failure, and patients with poor left ventricular ejection fraction (LVEF $<40 \%$ ) were also excluded.

\section{Management Regimen}

\section{Preoperatively}

The collected preoperative data from the study population were age, routine risk factors of cardiovascular disease e.g. hypertension, smoking, dyslipidemia, diabetes mellitus and family history of susceptibility to IHD, previous myocardial infarction (MI) and history of CCU admission, history of coronary angioplasty and stenting, chronic obstructive pulmonary disease (COPD), left ventricular ejection fraction (LVEF), EuroSCORE II, history of chronic renal disease, strok, peripheral vascular disease and vascular surgeries, carotid and peripheral arterial and venous duplex and routine laboratory preoperative investigations. Diabetic patients (regardless their preoperative glycosylated haemoglobin "HbA1c" level) were subjected to strict (tight) glycemic control measures using different doses of insulin therapy (individualized based on endocrinological consultations) aiming to keep blood glucose measures $110-150 \mathrm{mg} / \mathrm{dl}$. This included combination of scheduled subcutaneous insulin therapy and repeated insulin injections according to Matias protocol [16]. HCY level was measured and accordingly the two groups were formulated. Venous blood sample of $5 \mathrm{ml}$ was collected after an overnight fasting of 12-14 hours. Blood samples for the quantitation of plasma HCY were drown in EDTA (Ethylene Diamine Tetra Acetate) container tubes immediately placed in ice and centrifuged within 30-60 minutes for 10 minutes. The plasma separated and stored at $-20^{\circ} \mathrm{C}$ until analyzed. Total plasma $\mathrm{HCY}$ levels were determined using an immunofluorescence method (AXSYM, Abbott) already validated for this type of analysis [17] and applied in a previous large randomized controlled trial $[13,18]$. HCY concentrations were measured by ELISA (Enzyme Linked Immuno-Sorbent Assay) kits ( $\mathrm{HCY}$ immulite 2000, kit lot 264). Acetylsalicylic acid was discontinued 5 days before surgery while clexane and clopidogril were discontinued 12 hours and 5-7 days respectively before it. Angiotensin-converting enzyme inhibitors and angiotensin- 2 receptor blockers were discontinued at the night of surgery. All patients received sedative pre-medications (oral valium $5 \mathrm{mg}$ at the night of surgery and intramuscular morphia $10 \mathrm{mg}$ at the morning of surgery). The mortality operative risk was determined according to the logistic EuroSCORE II and all the risk conditions were defined according to the EuroSCORE II definitions [13].

\section{Intraoperatively}

All patients were pre-medicated by intravenous midazolam $(0.1 \mathrm{mg} / \mathrm{kg})$ at the operating room, patients were monitored with five-lead electrocardiogram (leads II and V simultaneously), pulse oximetry, invasive arterial blood pressure using an arterial catheter connected to a pressure transducer, capnography, central venous catheter inserted in the internal jugular vein, nasopharyngeal temperature probe, urinary catheter and frequent arterial blood gases (ABGs) measurements for $\mathrm{pH}$, electrolytes and glucose every 15 minutes. Di- abetic patients were subjected to intra-operative tight (strict) glycemic control using uniform intravenous insulin infusion protocol (prepared by mixing 100 units of insulin with $50 \mathrm{ml}$ $0.9 \%$ Normal Saline) aiming to keep blood glucose measures $110-150 \mathrm{mg} / \mathrm{dl}$. After pre-oxygenation, general anesthesia was induced with thiopental (3-5 mg/kg), Fentanyl (2-10 mg/ $\mathrm{kg}$ ) and pancuronium $(0.1 \mathrm{mg} / \mathrm{kg})$. Patients were then ventilated manually with face mask and intubated with an oral cuffed endotracheal tube with the proper diameter, followed by the onset of controlled mechanical ventilation. Anesthesia was maintained with isoflurane and additional doses of propofol infusion, fentanyl (1-2 micro.gm $/ \mathrm{kg})$ and pancuronium $(0.01 \mathrm{mg} / \mathrm{kg})$. Anticoagulation was established with an initial dose of heparin $(300-400 \mathrm{IU} / \mathrm{kg})$ and to get activated clotting time (ACT) higher than $400 \mathrm{~s}$, additional heparin was given on need to maintain ACT higher than $400 \mathrm{~s}$ during bypass time. At the end of CPB, heparin was reversed by protamine chloride at a 1:1 ratio of the loading dose, regardless of the total heparin dosage. Operative technique was always the same for all the study population. All the patients were operated upon via standard vertical median sternotomy, CPB was initiated with aorto-caval cannulation, ascending aorta was cross clamped and cardioplegia was received by warm blood intermittent antegrade technique every 20 minutes. The priming volume was always minimized to $800-1000 \mathrm{~mL}$. Core body temperature during CPB was $30-32{ }^{\circ} \mathrm{C}$. The pump flow was targeted between 2.0 and $2.8 \mathrm{~L} / \mathrm{min} / \mathrm{m}^{2}$ and the target mean arterial pressure was settled at $60 \mathrm{mmHg}$. Pedicled left internal thoracic (mammary) artery (LIMA) was always anastomosed to left anterior descending coronary artery (LAD) and the harvested reversed saphenous vein grafts (SVGs) were anastomosed to other target coronaries. Proximal anastmoses were carried on beating heart after instillation of hot shot dose and unclamping the ascending aorta regaining myocardial activity using partial aortic side occlusion clamping. The operative variables included intraoperative mortality, aortic cross clamping time, CPB time, number of grafts done, blood glucose level (during and after CPB), metabolic acidosis, haemodynamics, difficulty of weaning off CPB, inotropic support medications, need for intra-aortic balloon pump (IABP) insertion.

\section{Postoperatively}

Patients were shifted to the Intensive Care Unit (ICU) for stabilization prior to transfer to regular room. Patients were followed-up for one year postoperatively. The postoperative studied variables included ICU parameters (duration of mechanical ventilation, duration of inotropic support, total blood loss, blood glucose level, total duration of ICU stay), operative mortality, total hospital stay, mortality within one-year follow-up, and adverse complications (myocardial infarction, cerebrovascular accidents, pulmonary embolism, peripheral arterial/venous thromboembolism, low cardiac output syndrome, rhythmic complications, hemorrhagic complications, respiratory complications, acute renal failure, deep and superficial wound infections). The overall hospital complication rate was calculated on the basis of the number of patients with at least one hospital complication. 
Citation: Saber A, Fathy A, Nasr S (2019) Association of Hyperhomocysteinemia and Adverse Postoperative Outcomes of on-Pump Primary Isolated Coronary Artery Bypass Grafting Surgery among Postmenopausal Women. Ann Heart 4(1):86-95

\section{Definitions of Adverse Variables}

Perioperative myocardial infarction was defined as creatinine kinase-MB elevation of 5 or more times the upper limit of normal and the presence of any new $Q$ wave within 48 hours postoperatively or the disappearance of the $\mathrm{R}$ wave on the postoperative electrocardiogram (ECG). Cerebrovascular accident (CVA) was defined as a new stroke or a transient ischemic attack (TIA) for at least 24 hours. Low cardiac output syndrome was defined as the need for the use of two catecholamines beyond $10 \mathrm{microgram} / \mathrm{Kg} / \mathrm{min}$ dose or the use of an intra-aortic balloon pump (IABP). Operative mortality was defined as any death occurring during the 30 days postoperatively. Rhythmic complications were defined by the presence of a supraventricular or ventricular rhythm disorder. Hemorrhagic complications were defined by re-operation to control bleeding or to relieve cardiac tamponade. Respiratory complications were defined by the development of pneumonia, pulmonary atelectasis, and respiratory failure (prolonged ventilation more than 48 hours postoperatively, re-intubation or tracheostomy). Acute renal failure was defined as a rise in the creatinine level (absolute $\geq 0.3 \mathrm{mg} / \mathrm{dl}$, percentage $\geq 50 \%$ ) in need for renal replacement therapy or the need for dialysis sessions (excluding patients requiring dialysis before the operation). Preoperative chronic renal failure was defined as a creatinine clearance of less than $30 \mathrm{ml} / \mathrm{min}$. Preoperative peripheral vascular disease was defined as the presence of lower limb arterial disease stage I or II according to Leriche and Fontaine classification or a history of vascular surgery. Deep surgical wounds (sternal and lower limbs) infections were defined as a surgical infection occurring within 30 days, extending beyond the deep tissue plane with bacteriological samples of positive infected tissues or purulent discharge. Thromboembolic event was defined as the occurrence of any of: Myocardial infarction, stroke or TIA, pulmonary or systemic embolism. Major morbidity was defined as any of the following: need for re-operation, sternal wound infection, permanent stroke, renal failure, mechanical ventilation for more than 48 hours.

\section{Statistical Analysis}

All patients' data were tabulated and processed using SPSS V13.0 (SPSS Inc., Chicago, IL) for Windows 2007. Quantitative variables were expressed using mean and standard deviation, and were compared using $t$-student test. Qualitative variables were compared using Chi-square test or Fischer's exact test when appropriate. Correlation between parameters was performed using Spearman's rank correlation coefficient. In all tests, $P$ value was considered significant when $P<0.05$, highly significant when $P<0.01$ and extremely significant when $P<$ 0.001 .

\section{Results}

In the period between January 2016 and January 2019, this study was conducted on 77 postmenopausal women undergoing on-pump primary isolated CABG. The study population was divided into two groups based on their HCY level. Group (I) included 34 patients with $\mathrm{HCY}$ values more than 15 micro.mol/L and group (II) included 43 patients with HCY values less than 15 micro.mol/L. The preoperative, operative, postoperative and one-year follow-up data of both groups were thoroughly studied and compared.

\section{Preoperative data}

The age of the study population ranged from $54-70$ years with a median age of 61.5 years. The mean age for group (I) patients was $64.34 \pm 5.81$ years and for group (II) $60.95 \pm 6.54$ years. All patients had undergone a thorough clinical evaluation which included proper history taking, general, vascular, chest and cardiac examination. Mean HCY level was $18.55 \pm$ $1.11 \mathrm{micro} . \mathrm{mol} / \mathrm{L}$ (range 15.50-23.6) for group (I) and 9.83 $\pm 1.03 \mathrm{micro} . \mathrm{mol} / \mathrm{L}$ (range 5.6-12.7) for group (II) representing high statistical significance $(p<0.05)$. Group (I) showed statistically significant differences $(p<0.05)$ regarding the following variables: More previous $\mathrm{MI}$ attacks, more CCU admissions, lower LVEF\%, higher EuroSCORE, more peripheral vascular disease and surgeries, more previous cerebrovascular accidents, more number of affected coronary vessels and

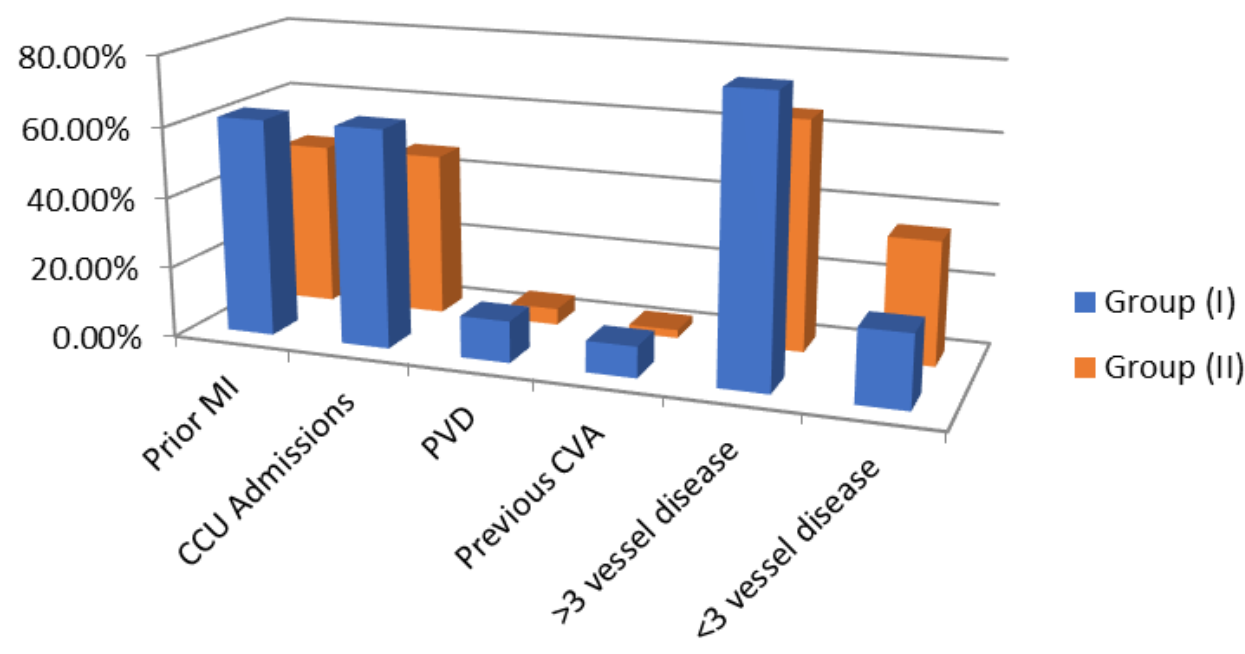

Figure 1: Some significant preoperative variables. 
Citation: Saber A, Fathy A, Nasr S (2019) Association of Hyperhomocysteinemia and Adverse Postoperative Outcomes of on-Pump Primary Isolated Coronary Artery Bypass Grafting Surgery among Postmenopausal Women. Ann Heart 4(1):86-95

higher serum creatinine levels) Figure 1. No patient of either group needed preoperative intra-aortic balloon insertion. Further analysis of the preoperative patients characteristics and laboratory investigations of both groups are summarized in Table 1.

\section{Operative data}

All patients of both groups were submitted for on-pump isolated primary CABG using the same technique of graft anastmosis with pedicled LIMA to LAD and the reversed SVGs for other coronary artery targets with the help of heart-lung ma-

Table 1: Preoperative patients characteristics and laboratory investigations.

\begin{tabular}{|c|c|c|c|c|}
\hline & $\begin{array}{l}\text { Group (I) } \\
(\mathrm{n}=34)\end{array}$ & $\begin{array}{l}\text { Group (II) } \\
(n=43)\end{array}$ & $P$ value & Significance \\
\hline Age (years) & $64.34 \pm 5.81$ & $60.95 \pm 6.54$ & $>0.05$ & NS \\
\hline HCY level (micro.mol/L) & $18.55 \pm 1.11(15.50-23.6)$ & $9.83 \pm 1.03(5.6-12.7)$ & $<0.05$ & Significant \\
\hline D.M. & $10(29.41 \%)$ & $13(30.23 \%)$ & $>0.05$ & NS \\
\hline Positive family history of IHD & $5(14.71 \%)$ & $7(17.28 \%)$ & $>0.05$ & NS \\
\hline Smoking & $0(0 \%)$ & $0(0 \%)$ & $>0.05$ & NS \\
\hline Hypertension & 22 (64.71\%) & 27 (62.79\%) & $>0.05$ & NS \\
\hline Dyslipidaemia & 27 (79.41\%) & $33(76.74 \%)$ & $>0.05$ & NS \\
\hline Previous MI & $21(61.76 \%)$ & $20(46.51 \%)$ & $<0.05$ & Significant \\
\hline History of CCU admission & $21(61.76 \%)$ & $20(46.51 \%)$ & $<0.05$ & Significant \\
\hline History of $\mathrm{PCl}$ and stenting & $11(32.35 \%)$ & $16(37.21 \%)$ & $>0.05$ & NS \\
\hline LVEF \% & $52.33 \pm 4.12$ & $59.71 \pm 3.35$ & $<0.05$ & Significant \\
\hline EuroSCORE II & $4(2-7)$ & $3(2-5)$ & $<0.05$ & Significant \\
\hline COPD & $4(11.76 \%)$ & $3(6.98 \%)$ & $>0.05$ & NS \\
\hline Chronic renal disease & $1(2.94 \%)$ & $2(4.65 \%)$ & $>0.05$ & NS \\
\hline Peripheral vascular disease & $4(11.76 \%)$ & $2(4.65 \%)$ & $<0.05$ & Significant \\
\hline Previous CVA & $3(8.82 \%)$ & $1(2.32 \%)$ & $<0.05$ & Significant \\
\hline LM or LM equivalent disease & $15(44.11 \%)$ & $18(41.86 \%)$ & $>0.05$ & NS \\
\hline 3 or more vessel disease & 27 (79.41\%) & $28(65.11 \%)$ & $>0.05$ & Significant \\
\hline Less than 3 vessel disease & $7(20.58 \%)$ & 15 (34.88\%) & $>0.05$ & Significant \\
\hline Peak serum creatinine $(\mathrm{mg} / \mathrm{dl})$ & $1.36 \pm 0.29$ & $0.90 \pm 0.35$ & $>0.05$ & Significant \\
\hline Urea & $52.3 \pm 5.82$ & $42.3 \pm 7.13$ & $>0.05$ & NS \\
\hline FBG (on admission) & $217.8 \pm 21.1$ & $223.3 \pm 14.1$ & $>0.05$ & NS \\
\hline FBG (just prior to surgery) & $139.8 \pm 31.7$ & $142.6 \pm 15.3$ & $>0.05$ & NS \\
\hline
\end{tabular}

(Data were expressed as mean \pm standard deviation (SD) or number (\%), NS = Not Significant).

Table 2: Operative data.

\begin{tabular}{|c|c|c|c|c|}
\hline & $\begin{array}{l}\text { Group (I) } \\
(n=34)\end{array}$ & $\begin{array}{l}\text { Group (II) } \\
(n=43)\end{array}$ & $P$ value & Significance \\
\hline Total operation time (min) & $188.53 \pm 28.41$ & $190.23 \pm 30.11$ & $>0.05$ & NS \\
\hline Total CPB time (min) & $100.27 \pm 21.35$ & $100.29 \pm 21.72$ & $>0.05$ & NS \\
\hline Total cross clamp time (min) & $61.54 \pm 11.39$ & $61.42 \pm 36.89$ & $>0.05$ & NS \\
\hline LIMA-to-LAD anastmosis & $34(100 \%)$ & $43(100 \%)$ & $>0.05$ & NS \\
\hline 3 or more coronary targets & $27(79.41 \%)$ & $28(65.11 \%)$ & $<0.05$ & Significant \\
\hline Less than 3 coronary targets & $7(20.58 \%)$ & $15(34.88 \%)$ & $<0.05$ & Significant \\
\hline Metabolic Acidosis & $9(26.47 \%)$ & $12(27.91 \%)$ & $>0.05$ & NS \\
\hline Glucose level (during CPB) (mg/dl) & $303.98 \pm 41.19$ & $302.76 \pm 15.37$ & $>0.05$ & NS \\
\hline Glucose level (after CPB) (mg/dl) & $210.5 \pm 21.8$ & $221.6 \pm 36.1$ & $>0.05$ & NS \\
\hline Smooth weaning off-CPB & $29(85.29 \%)$ & $37(86.04 \%)$ & $>0.05$ & NS \\
\hline
\end{tabular}

(Data were expressed as mean \pm standard deviation (SD) or number (\%), NS = Not Significant). 
Citation: Saber A, Fathy A, Nasr S (2019) Association of Hyperhomocysteinemia and Adverse Postoperative Outcomes of on-Pump Primary Isolated Coronary Artery Bypass Grafting Surgery among Postmenopausal Women. Ann Heart 4(1):86-95

chine and antegrade warm blood intermittent cardioplgia given every 20 minutes. Serial arterial blood samples were taken every 15 minutes. No intraoperative mortality happened as well as no intra-aortic balloon was needed to assist haemodynamics. Metabolic acidosis was encountered and continuously corrected in $26.47 \%$ and $27.91 \%$ of group (I) and (II) respectively. However, it was controlled before transferring patients to ICU and no one faced persistant acidosis. All patients were shifted to ICU on minimal inotropic support. Haemodynamic parameters during the operation were recorded showing no significant differences among both groups. Smooth weaning off-CPB was achieved in 29 (85.29\%) of group (I) and 37 (86.04\%) of group (II) $(p>0.05)$. The rest of the patients needed electrical cardioversion to achieve weaning. The date of the operation was recorded and the operative reports for both groups were assessed and the data recorded, analysed and compared. The data are shown in Table 2.

\section{Postoperative data}

All the patients were discharged to the ICU mechanically ventilated. ABGs were obtained from arterial blood samples every 2 hours. Patients were discharged from the ICU when haemodynamically stable on no inotropic support, with no drains and with satisfactory postoperative laboratory results and ECG. The mean duration of mechanical ventilation for group (I) was $13.20 \pm 2.31$ hours compared to $6.1 \pm 2.58$ hours for group (II) $(p<0.05)$. The mean period of the total ICU stay was $59.51 \pm 1.12$ hours for group (I) and $46.34 \pm 3.45$ hours for group (II) patients $(p<0.05)$. The mean duration of the total hospital stay was $9.87 \pm 3.56$ days in group (I) and 6.17 \pm 1.52 days in group (II) $(p<0.05)$. There were a total of 2 (2.59\%) mortality cases, $1(2.94 \%)$ in group (I) and 1 (2.32\%) in group (II) $(p>0.05)$. All of them happened in the postoperative ICU period due to biventricular heart failure. Cerebrovascular accidents occurred in $4(11.76 \%)$ in group (I) compared to $1(2.32 \%)$ in group (II) $(p<0.05)$. Low cardiac output syndrome was faced and corrected in $3(8.82 \%)$ in group $(\mathrm{I})$ and $1(2.32 \%)$ in group (II) $(p<0.05)$. Statistically significant differences emerged between the study groups as regards the incidence of acute renal failure, peak serum creatinine values and the need of IABP insertion in the ICU Figure 2. However, no statistically significant differences were found regarding inotropic support duration, total blood loss, perioperative MI, rhythmic complications, haemorrhagic complications, deep surgical wound infection, superficial surgical wound infection and respiratory complications as been shown in Table 3. Moreover, the overall hospital complication rate was 12 (35.29\%) and $13(30.23 \%)$ for group (I) and (II) respectively ( $p$ $>0.05$ ). After hospital discharge, patients were followed-up for one year. Patients were called after a mean period of $349.07 \pm 22.15$ days in group (I) and $351.86 \pm 11.77$ days in group (II) $(p>0.05)$. The mean period for return to work was $54.21 \pm 9.91$ days in group (I) and $53.63 \pm 21.50$ days in group (II) $(p>0.05)$. Two more mortality cases happened during the follow-up period (one in each group). Thus, the overall mortality reached $5.19 \%$.

\section{Discussion}

$\mathrm{HHC}$ is caused by various causatives including deficiency of folic acid, vitamin B12 and vitamin B6, deficiency in enzymes like cystathione beta-synthase, methionine synthase, methylene tetrahydro-folate reductase, intake of methotrexate, phenitoin, nitrous oxide, theophylline, L-dopa, cholestramine, niacin, smoking, chronic alcoholism and coffee consumption $[1,5,19]$.

Affected patients with $\mathrm{HHC}$ have a high incidence of vascular pathology that may result in early death from myocardial infarction, stroke, or venous thromboembolism [20]. Prior investigations have shown that HHC is associated with an increased risk for cardiovascular disease, including coronary artery disease, peripheral arterial disease, stroke, and venous thrombosis [21,22]. Moreover, HHC has been associated with increased risk of morbidity and mortality in patients with established coronary artery or cerebrovascular disease [23]. Even modest increases in the concentration of HCY have been reported in patients with coronary artery disease, stroke, and peripheral vascular disease [19]. The

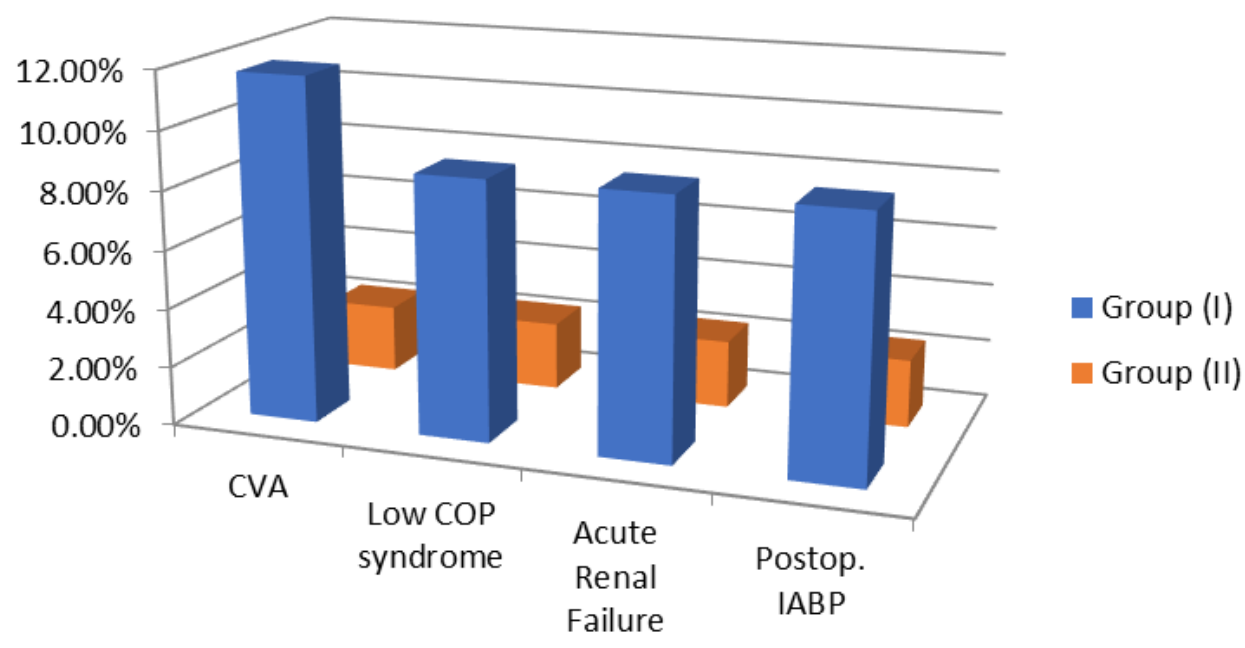

Figure 2: Some significant postoperative variables. 
Citation: Saber A, Fathy A, Nasr S (2019) Association of Hyperhomocysteinemia and Adverse Postoperative Outcomes of on-Pump Primary Isolated Coronary Artery Bypass Grafting Surgery among Postmenopausal Women. Ann Heart 4(1):86-95

Table 3: Postoperative data.

\begin{tabular}{|c|c|c|c|c|}
\hline & $\begin{array}{l}\text { Group (I) } \\
(\mathrm{n}=34)\end{array}$ & $\begin{array}{l}\text { Group (II) } \\
(n=43)\end{array}$ & $p$ value & Significance \\
\hline Mechanical ventilation time (hrs) & $13.20 \pm 2.31$ & $6.1 \pm 2.58$ & $<0.05$ & Significant \\
\hline Inotropic support (hrs) & $14.9 \pm 2.6$ & $11.8 \pm 3.5$ & $>0.05$ & NS \\
\hline ICU stay (hrs) & $59.51 \pm 1.12$ & $46.34 \pm 3.45$ & $<0.05$ & Significant \\
\hline Total blood loss (ml) & $332.5 \pm 415.1$ & $347.07 \pm 457.2$ & $>0.05$ & NS \\
\hline Glucose level (before ICU discharge) (mg/dl) & $190.5 \pm 10.6$ & $191.5 \pm 35.1$ & $>0.05$ & NS \\
\hline Glucose level (before hospital discharge) (mg/dl) & $139.2 \pm 27.7$ & $147.3 \pm 18.5$ & $>0.05$ & NS \\
\hline Peak serum creatinine $(\mathrm{mg} / \mathrm{dl})$ & $1.10 \pm 0.15$ & $0.70 \pm 0.22$ & $>0.05$ & Significant \\
\hline Perioperative MI & $2(5.88 \%)$ & $1(2.32 \%)$ & $>0.05$ & NS \\
\hline Low cardiac output syndrome & $3(8.82 \%)$ & $1(2.32 \%)$ & $<0.05$ & Significant \\
\hline Operative mortality & $1(2.94 \%)$ & $1(2.32 \%)$ & $>0.05$ & NS \\
\hline Stroke/TIAs & $4(11.76 \%)$ & $1(2.32 \%)$ & $<0.05$ & Significant \\
\hline Atrial Fibrillation & $3(8.82 \%)$ & $3(6.98 \%)$ & $>0.05$ & NS \\
\hline Ventricular arrhythmias & $4(11.76 \%)$ & $5(11.62 \%)$ & $>0.05$ & NS \\
\hline Haemorrhagic complications & $1(2.94 \%)$ & $1(2.32 \%)$ & $>0.05$ & NS \\
\hline Deep wound infection & $2(5.88 \%)$ & $3(6.98 \%)$ & $>0.05$ & NS \\
\hline Superficial wound infection & $2(5.88 \%)$ & $2(4.65 \%)$ & $>0.05$ & NS \\
\hline Respiratory complications & $5(14.70 \%)$ & $4(9.30 \%)$ & $>0.05$ & NS \\
\hline Acute renal failure & $3(8.82 \%)$ & $1(2.32 \%)$ & $<0.05$ & Significant \\
\hline Postoperative IABP insertion & $3(8.82 \%)$ & $1(2.32 \%)$ & $<0.05$ & Significant \\
\hline Total hospital stay (days) & $9.87 \pm 3.56$ & $6.17 \pm 1.52$ & $<0.05$ & Significant \\
\hline Overall hospital complications & 12 (35.29\%) & $13(30.23 \%)$ & $>0.05$ & NS \\
\hline Mortality in follow-up period & $1(2.94 \%)$ & $1(2.32 \%)$ & $>0.05$ & NS \\
\hline Overall one-year survival & 32 (94.11\%) & 41 (95.34\%) & $>0.05$ & NS \\
\hline
\end{tabular}

(Data were expressed as mean \pm standard deviation (SD) or number (\%), NS = Not Significant).

interaction of $\mathrm{HHC}$ with dyslipidemia, hypertension, diabetes and smoking is strong, and the combined effect of these factors is additive [24].

The possible mechanisms for HHC leading to the development of atherosclerosis may be due to various factors including endothelial cell damage, smooth muscle cell proliferation, thromboembolism, and inability to sustain S-nitrose-homocysteine (a potent vasodilator and inhibitor of platelet aggregation) formation because of imbalance between nitric oxide production by dysfunctional endothelium and $\operatorname{HCY}[1,25]$.

Plasma HCY is significantly lower in premenopausal women than young men but after menopause basal homocysteinemia increases significantly in women approaching those in men. It seems likely that altered hormonal status and age related low folate intake are responsible for this. For women, cardiovascular disease is largely a disease of post menopause. A woman's risk of heart disease is far lower than man's risk until after menopause. This change in incidence of heart disease may be related to advancing age, changes in hormonal milieu, or other unknown factors [26].

The association between homocystinuria, a genetic disorder with extremely high plasma HCY levels (in excess of 100
micro.mol/L), and premature atherosclerosis, increased risk of thromboembolism and premature death has been known for more than last five decades [13]. This lead to series of epidemiological investigations that have shown that even moderate elevation of $\mathrm{HCY}$ is associated with increased risk of coronary artery disease, peripheral arterial disease, stroke, and venous thromboembolic disease $[21,22]$.

Storti, et al. [27] studied the changes in HCY levels in a population of patients who underwent CABG. They detected a mean preoperative value $17.3 \mathrm{micro}$. mol/L comparable to $18.55 \pm 1.11$ micro.mol/L in our study and higher than the one mentioned in the study done by Ranucci, et al. [13] which was $15.4 \pm 8.3 \mathrm{micro} . \mathrm{mol} / \mathrm{L}$. They stated that HCY levels significantly decreased after the operation. However, no outcome analyses were performed in this study, which included 48 patients compared to 77 patients in our study who received meticulous analysis of postoperative outcomes. Ranucci, et al. [13] suggested heightened risk of perioperative morbidity and mortality in patients with elevated HCY levels undergoing surgical coronary revascularization. However, the previously quoted studies suggested that in patients with cardiovascular disease, elevated $\mathrm{HCY}$ levels confer heightened risk of adverse events over intermediate or long-term follow-up [13,21-23]. 
Citation: Saber A, Fathy A, Nasr S (2019) Association of Hyperhomocysteinemia and Adverse Postoperative Outcomes of on-Pump Primary Isolated Coronary Artery Bypass Grafting Surgery among Postmenopausal Women. Ann Heart 4(1):86-95

These results meet ours as regards our findings of significant preoperative variables (more previous $\mathrm{MI}$ attacks, more CCU admissions, lower LVEF\%, higher EuroSCORE, more peripheral vascular disease and surgeries, more previous cerebrovascular accidents, more number of affected coronary vessels and higher peak serum creatinine levels) and postoperative outcomes (low cardiac output syndrome, need for IABP insertion, more cerebrovascular accidents, acute renal failure and higher peak serum creatinine level). These adversely impacting outcomes were reflected on the prolonged duration of postoperative mechanical ventilation, period of ICU stay and total period of hospital stay although the overall hospital complication rate was 12 (35.29\%) and 13 (30.23\%) for group (I) and (II) respectively $(p>0.05)$. During the follow-up period, both groups expressed comparable results with no statistical significance, the overall one-year survival was $94.11 \%$ and $95.34 \%$ in group (I) and (II) respectively ( $p>0.05)$ and the overall mortality was 4 (5.19\%) (two deaths from each group; one in-hospital, and one during the follow-up period) $(p>0.05)$. Thus, operative and postoperative mortality was found not to be associated with HHC.

Our results confer and emphasize the association of $\mathrm{HHC}$ with the postoperative adverse events particularly thromboembolic ones as has been stated before [23]. The experimental hypothesis was that patients with $\mathrm{HHC}$ would have a higher rate of post-operative thromboembolic events than patients without $\mathrm{HHC}$ and the rate of thromboembolic events in patients with $\mathrm{HHC}$ was assumed to be twice that in those without HHC [13]. Ranucci, et al. [13] mentioned collectively $7.4 \%$ incidence of thromboembolic events (myocardial infarction, stroke, mesenteric infarction, pulmonary and peripheral embolism) compared to ours $8.82-11.76 \%$. This is not surprising if we consider the hypothesis on the mechanisms underlying the association of $\mathrm{HHC}$ with adverse outcomes in patients with cardiovascular disease where high level of HCY impairs endothelial function and decreases the bioavailability of nitric oxide from endothelial cells. It also reduces the affinity of the anticoagulant antithrombin to bind to endothelial cells [28] and it may limit the coagulation inhibitory effect normally exerted by the thrombomodulin-protein $\mathrm{C}$ complex through a reduction in protein $\mathrm{C}$ activation rate [28]. These mechanisms decrease endothelial resistance to thrombosis, reduce vasodilatory effects of nitric oxide, and promote thrombosis. Thus, on-pump CABG operations with CPB which itself leads to the hypercoaguable state by the generation of massive doses of thrombin that consumes antithrombin [29] by decreasing nitric oxide synthesis $[29,30]$ and by reducing protein $C$ and tissue factor pathway inhibitor [31]. Therefore, it seems reasonable why $\mathrm{HHC}$ may further aggravate the hypercoaguable state, thereby increasing the thromboembolic risk in these patients. These mechanisms are supported by the fact that the great majority of perioperative complications observed in patients with $\mathrm{HHC}$ undergoing CABG seem to be thromboembolic events resulting from either macrovascular clots in cerebral circulation or microvascular thrombosis in coronary and renal microcirculation (low cardiac output and acute renal failure) accounting for $9 \%$ probability increase for each 1 micro.mol/L increment [13].
Ranucci, et al. [13] found that the increased risk of adverse events was only partially explained by older age and greater comorbid conditions such as lower left ventricular ejection fraction and lower creatinine clearance (accounting for their higher EuroSCORE) but HHC remained an independent correlate of higher need for blood transfusion, acute renal failure, low cardiac output syndrome, any thromboembolic event, but more importantly, in-hospital mortality. The association of elevated $\mathrm{HCY}$ level with mortality was observed predominantly among patients undergoing CABG, but not in patients undergoing isolated valve surgery. However, they stated that preoperative HCY levels have an acceptable predictive ability for thromboembolic events, but lower for in-hospital mortality. Our study group population was younger. The mean age was $64.34 \pm 5.81$ years in group (I) and 60.95 \pm 6.54 years in group (II) compared to 71 (61-77) years in their study. The mean LVEF\% was $52.33 \pm 4.12$ and $59.71 \pm 3.35$ for group (I) and (II) respectively compared to 50 (38-57)\% in their study. The mean peak serum creatinine $(\mathrm{mg} / \mathrm{dl})$ was $1.36 \pm 0.29$ and $0.90 \pm 0.35$ for group (I) and (II) respectively compared to 1.2 (1-1.6) in their study. Group (I) had EuroSCORE 4 (2-7) and group (II) had 3 (2-5) compared to 6 (3-10) in their study. Thus, our study group expressed younger age, better LVEF\% and EuroSCORE and still HHC was associated with adverse postoperative outcomes. However, it was not associated to the previously claimed operative and postoperative mortality and did not influence one-year survival.

The prevalence of $\mathrm{HHC}$ in the pre-CABG population is unknown. However, it is reasonable to assume it to be higher in patients with cardiovascular disease than the $11 \%$ prevalence of HHC described in the European population [18]. The study by Ranucci, et al. [13] suggested that the prevalence of pre-operative $\mathrm{HHC}$ is high, with two of every five patients undergoing cardiac surgery having elevated $\mathrm{HCY}$ values more than 15 micro.mol/L. They assumed a prevalence of $30 \%$ in their patient population which included ischemic and valvular cardiac operations. We found a prevalence of $44.15 \%$ among our IHD postmenopausal women; a figure that seems to be more fitting and representative particularly for this subset cohort.

Folic acid, alone or combined with other B vitamins, is safe and effective in lowering plasma HCY levels [32]. It is required for $\mathrm{HCY}$ metabolism and folate deficiency is associated with elevated $\mathrm{HCY}$ concentrations. Thus, supplementation of folic acid reduces HCY levels in normal subjects and in patients at risk for cardiovascular disease $[33,34]$. It is well known that vitamin supplementation lowers HCY concentrations and reverses endothelial dysfunction in almost all subjects with $\mathrm{HHC}$, regardless of the underlying cause $[1,24]$.

The observation of an association between a raised plasma homocysteine level and the occurrence of coronary atherosclerosis may imply the need for greater efforts to correct the nutritional factors that affect HCY metabolism [19]. Our study has shown that HHC is associated with IHD and it is a risk factor for coronary atherosclerosis. Thus, we suggest that preoperative ischemic postmenopausal ladies with HHC should be treated with folic acid (2-5 mg/day) [33,34], vitamin $\mathrm{B} 12$, and vitamin B6. Moreover, the factors that may raise the 
Citation: Saber A, Fathy A, Nasr S (2019) Association of Hyperhomocysteinemia and Adverse Postoperative Outcomes of on-Pump Primary Isolated Coronary Artery Bypass Grafting Surgery among Postmenopausal Women. Ann Heart 4(1):86-95

serum HCY level such as use of certain drugs, smoking, chronic alcohol and coffee consumption should be avoided. However, treating patients who have $\mathrm{HHC}$ with these vitamins has failed to decrease cardiovascular morbidity and mortality in two completed large randomized controlled trials $[18,35]$. Thus, this high-risk group of perioperative thromboembolic events could be offered other surgical approach without CBP (off-pump CABG) or pharmacological supplementation with antithrombin may be beneficial in improving outcomes $[29,36]$.

\section{Conclusion}

HHC was found to have high prevalence among postmenopausal women having IHD and undergoing CABG surgery. It was found to be associated with adverse postoperative complications as well as preoperative thromboembolic and cardiovascular insults. On-pump CABG aggravates the thrombotic potentiality in these patients. However, operative and postoperative mortality as well as one-year survival were not associated with $\mathrm{HHC}$. Preoperative assay of $\mathrm{HCY}$ level and $\mathrm{HHC}$ stratification in the reviewed pre-CABG risk factors are recommended. Preoperative supplementation with folic acid, vitamin B12 and vitamin B6 may play a role in lowering the preoperative HCY level, thus, lowering the proposed postoperative adverse outcomes. Therapeutic strategies such as preoperative aggressive treatment with antiplatelet or anticoagulant drugs may also be tried to reduce the thrombogenic risks for this subset of patients. This study may raise attention for further researches to HHC; a variable that has been associated with postoperative complications.

\section{Limitations}

The limitations of our study were the reduced number of the study population and the short-term one-year followup. Effect of HHC on intermediate and long-term outcomes was not involved in the study. Our results were confined to the postmenopausal women undergoing elective on-pump isolated CABG. Thus, male sex, combined CABG, off-pump $C A B G$, urgent/emergent $C A B G$ and valvular surgeries were not in the scope of our study.

\section{References}

1. Prasad K (1999) Homocysteine, a risk factor for cardiovascular disease. Int J Angiol 8: 76-86.

2. de Bree A, van der Put NM, Mennen LI, et al. (205) Prevalences of hyperhomocysteinemia, unfavorable cholesterol profile and hypertension in European populations. Eur J Clin Nutr 59: 480-488.

3. Eikelboom JW, Lonn E, Genest J Jr, et al. (1999) Homocysteine and cardiovascular disease: A critical review of the epidemiologic evidence. Ann Intern Med 131: 363-375.

4. Smith SC, Greenland P, Grundy SM (2000) AHA Conference proceedings. Prevention conference $\mathrm{V}$ : Beyond secondary prevention: Identifying the high-risk patient for primary prevention: executive summary. American Heart Association. Circulation 101: 111-116.

5. Li J, Zhang Y, Yao X, et al. (2002) Effect of homocysteine on the L-arginine/nitric oxide synthase/nitric oxide pathway in human platelets. Heart Vessels 16: 46-50.
6. Bozkurt A, Toyaksi H, Acarturk E, et al. (2003) The effects of hyperhomocysteinemia on the presence, extent, and severity of coronary artery disease. Jpn Heart J 44: 357-368.

7. Hughes $S$ (2003) Novel cardiovascular risk factors. J Cardiovasc Nurs 18: 131-138.

8. Lentz SR (2005) Mechanisms of homocysteine-induced atherothrombosis. J Thromb Haemost 3: 1646-1654.

9. Reiss RP, Azinheira J, Reiss HP, et al. (1999) The effect of sex and menopause on basal blood levels of homocysteine and after methionine loading. Rev Port Cardiol 18: 155-159.

10. Knopp RH (2002) Risk factors for coronary artery disease in women. Am J Cardiol 89: 34-35.

11. Fernandez-Miranda C, De la Calle M, Manuel Bris J, et al. (2001) Influence of menopausal status in homocysteine plasma levels. Med Clin Barc 116: 206-208.

12. Verhoef $P$ (2000) Hyperhomocysteinemia and risk of vascular disease in women. Semin Thromb Hemost 26: 325-334.

13. Marco Ranucci, Andrea Ballotta, Alessandro Frigiola, et al. (2009) Pre-operative homocysteine levels and morbidity and mortality following cardiac surgery. Eur Heart J 30: 995-1004.

14. The PREVENT IV Investigators, Alexander JH, Hafley G, et al. (2005) Efficacy and safety of edifoligide, an E2F transcription factor decoy, for prevention of vein graft failure following coronary artery bypass graft surgery-PREVENT IV: A randomized controlled trial. JAMA 294: 2446-2454.

15. Anyanwu AC, Filsoufi F, Salzberg SP, et al. (2007) Epidemiology of stroke after cardiac surgery in the current era. J Thorac Cardiovasc Surg 134: 1121-1127.

16. J Blaha, P Kopecky, M Matias, et al. (2009) Comparison of three protocols for tight glycemic control in cardiac surgery patients. Diabetes Care 32: 757-761.

17. Brunelli T, Pepe G, Marcucci R, et al. (2001) Comparison of three methods for total homocysteine plasma determination. Clin Lab 47: 393-397.

18. The Heart Outcomes Prevention Evaluation (HOPE) 2 Investigators (2006) Homocysteine lowering with folic acid and B vitamins in vascular disease. N Engl J Med 354: 1567-1577.

19. Necip Becit, Sait Keles, Yahya Ünlü, et al. (2005) Homocysteine Levels in Patients Undergoing Open-Heart Surgery. The Heart Surgery Forum 8.

20. Malinow MR, Bostom AG, Krauss RM (1999) Homocysteine, diet, and cardiovascular diseases: a statement for healthcare professionals from the Nutrition Committee, American Heart Association. Circulation 99: 178-182.

21. The Homocysteine Studies Collaboration (2002) Homocysteine and risk of ischemic heart disease and stroke: A meta-analysis. JAMA 288: 2015-2022.

22. Ford ES, Smith SJ, Stroup DF, et al. (2002) Homocysteine and cardiovascular disease: A systematic review of the evidence with special emphasis on case-control studies and nested casecontrol studies. Int J Epidemiol 31: 59-70.

23. Stubbs PJ, Al-Obaidi MK, Conroy RM, et al. (2000) Effect of plasma homocysteine concentration on early and late events in patients with acute coronary syndromes. Circulation 102: 605-610.

24. Hackam DG, Peterson JC, Spence JD (2000) What level of plasma homocyst(e)ine should be treated? Effects of vitamin therapy 
on progression of carotid atherosclerosis in patients with homocysteine levels above and below $14 \mathrm{imol} / \mathrm{L}$. Am J Hypertens 13: $105-110$.

25. Milosevic-Tosic M, Borota J (2002) Hyperhomocysteinemia: A risk factor for development of occlusive vascular diseases. Med Pregl 55: 385-391.

26. Ronald, Gibbs, Bethy, et al. (2008) Danforth's obstetrics and Gynecology. (10 edn), Lippincott Williams \& Wilkins, 735-736.

27. Storti S, Cerrillo AG, Rizza A, et al. (2004) Coronary artery bypass grafting surgery is associated with a marked reduction in serum homocysteine and folate levels in the early postoperative period. Eur J Cardiothorac Surg 26: 682-686.

28. Nishinaga M, Ozawa T, Shimada K (1993) Homocysteine, a thrombogenic agent, suppresses heparan sulfate expression in cultured porcine aortic endothelial cells. J Clin Invest 92: 13811386.

29. Ranucci M, Frigiola A, Menicanti L, et al. (2005) Postoperative antithrombin levels and outcome in cardiac operations. Crit Care Med 33: 355-360.

30. Ranucci M, Ditta A, Boncilli A, et al. (2004) Determinants of antithrombin consumption in cardiac operations requiring cardiopulmonary bypass. Perfusion 19: 47-52.
31. Boyle EM Jr, Morgan EN, Verrier ED (2000) The endothelium disturbed: The procoagulant response. In: Spiess BD, The relationship between coagulation, inflammation and Endothelium-a pyramid towards outcome. Lippincot Williams and Wilkins, Baltimore, Maryland, 79-89.

32. Lobo A, Naso A, Arheart K, et al. (1999) Reduction of homocysteine levels in coronary artery disease by low-dose folic acid combined with vitamins B6 and B12. Am J Cardiol 83: 821-825.

33. Mohamed Elkadi, Ayatt F Farag (2013) The effect of folic acid supplementation on serum homocysteine of Egyptian postmenopausal women. Middle East Fertility Society Journal 19.

34. Nasrolahi S, Radnia N, Neghab N, et al. (2010) Effect of Lowdose Folic acid Supplementation versus HRT and Combination of folic acid and HRT on Plasma Level of Homocysteine in Postmenopausal Women. Pak J Med Sci 26: 310-313.

35. Bønaa KH, Njølstad I, Ueland PM, et al. (2006) Homocysteine lowering and cardiovascular events after acute myocardial infarction. N Engl J Med 354: 1578-1588.

36. Koster A, Chew D, Kuebler W, et al. (2003) High antithrombin III levels attenuate hemostatic activation and leukocyte activation during cardiopulmonary bypass. J Thorac Cardiovasc Surg 126: 906-907. 